\title{
Self-Esteem Conditioning for Learning Conditioning
}

\author{
Imène Jraidi, Maher Chaouachi, Claude Frasson \\ HERON Lab; Computer Science Department \\ University of Montreal, CP 6128 succ. Centre Ville \\ Montréal, QC, H3T-1J4, Canada \\ \{jraidiim, chaouacm, frasson\}@iro.umonteal.ca
}

\begin{abstract}
In this paper, we propose to introduce the selfesteem component within learning process. More precisely, we explore the effects of learner self-esteem conditioning in a tutoring system. Our approach is based on a subliminal priming method aiming at enhancing implicit self-esteem. An experiment was conducted while participants were outfitted with biofeedback device. Three physiological sensors were used to continuously monitor learners' affective reactions namely electroencephalogram, skin conductance and blood volume pulse sensors. The purpose of this work is to analyze the impact of self-esteem conditioning on learning performance on one hand and learners' emotional and mental states on the other hand.
\end{abstract}

Subliminal priming; self-esteem; learning performance; sensor; learner affect

\section{INTRODUCTION}

Ongoing development in the field of affect recognition and modeling is providing Intelligent Tutoring Systems (ITS) with the possibility to integrate an emotional dimension while interacting with learners. Many researches use a variety of physical cues to detect emotional state [1] including observable changes like face expressions, body postures, vocal tones, and physiological signal changes such as heart rate, skin conductivity, temperature, and respiration. Other systems monitor brain electrical activity to assess learners' mental states [2].

Indeed, affect is seen as a driver of learning [3] and ITS seek to adapt their tutorial interventions according to learners' mental and emotional states identifying learners' emotions [4], or detecting stress and frustration [5].

Another important aspect in learning, advocated by pedagogues and educators, is learner self-esteem. A broad strand of research investigated the positive effects of selfesteem on learner self-confidence [6]. Besides, several studies have shown strong correlations between selfesteem and academic achievement and success [7].

Recently, McQuiggan, Mott, and Lester [8] proposed an inductive approach to model learner self-efficacy. They used learners' demographic and physiological data to predict their self-efficacy level. While self-efficacy represents the individual's belief about her ability to execute specific tasks, self-esteem is a more generalized aspect [9]. It reflects the overall personal self evaluation. Literature distinguishes between explicit self-esteem and implicit self-esteem [10]. The first is based on conscious mode of thinking and can be measured by means of questionnaires, whereas the latter is the result of automatic self-evaluative process and can be assessed with indirect measures. Unlike explicit measures which are based on generally biased self-report, implicit measures are based on unconscious attitude toward the self [10].

These latter measures are typically used in unconscious process based researches, more precisely, in the neuro-psychological communities. The core of these researches is the existence of a threshold-line of conscious perception. The idea is that a stimulus below this threshold of awareness, also called subliminal stimulus cannot be consciously perceived but can yield affective reactions without awareness [11]. Known as subliminal priming, this technique has been applied in different contexts [12] including self-esteem conditioning and learning improvement.

In this paper, we address the following issue: can implicit self-esteem conditioning produce a positive effect on learning? In this study, we conducted an experiment using a subliminal priming strategy in order to condition learner self-esteem while interacting with a tutoring system. Participants were outfitted with biofeedback device to continuously monitor their physiological activity. Electroencephalogram, skin conductance and blood volume pulse sensors were used. Our purpose was to analyze the effects of the subliminal priming strategy on learning performance and learners' affective states.

This paper is organized as follows. In the first section, we present previous works on the subliminal priming approach. In the second section, we describe the experimental environment and methodology. In the third section, we discuss the obtained results, conclude and present directions for future work.

\section{PREVIOUS WORK}

Researches devoted to automatic or unconscious processes have increased over the last years. Their basic assumption lies on the existence of a threshold-line between conscious and unconscious perception [11]. A stimulus is known as subliminal, if it is received below this threshold of awareness and cannot be consciously reported. High-level semantic and even emotional 
processing has been observed during this stage [12]. Masked priming is one of the main techniques used to project subliminal information [11]. In this method, the subliminal stimulus, also called prime, is projected during very short time. The prime is preceded and/or followed by the projection of a mask for a specific time. This mask usually takes the form of a series of symbols having nothing to do with the prime in order to elude its conscious detection.

In the Human Computer Interaction (HCI) community Wallace, Flanery and Knezek [13] implemented subliminal clues for task-supported operation within a text editor program. They found that the frequency at which subjects demanded help was much lower when the required information was subliminally presented. In another perspective, DeVaul, Pentland and Corey [14] used subliminal clues for just-in time memory support. They investigated the effect of various subliminal information on retention in a word-face learning paradigm.

In the ITS community, Chalfoun and Frasson [15] used a subliminal priming method within a $3 \mathrm{D}$ virtual tutoring system. It was found that overall performance was better, and time for answering questions was shorter for learners primed with subliminal clues. Learners' emotional reactions were also different; subliminal stimuli elicited high arousal states. Thus, besides yielding better results, subliminal priming seemed to elicit emotional consequences not only in learning, but also in various other domains like: social behavior, advertisement, stereotypes, food preferences, etc. (see [12] for a review). On the other side, evidence from this body of literature indicates that this effect is more important compared to consciously perceived and reported stimulus effects [16].

A recent work of Radel, and colleagues [17] put forward an interesting effect that subliminal priming can have on motivational processes. They investigated the impact of motivational primes in a natural setting, namely the classroom. A positive effect of subliminal priming on academic performance was found; this effect was basically moderated by learners' mindfulness.

In this paper, we propose to introduce a new approach to subliminally enhance learner self-esteem while interacting with a tutoring system. We are interested in analyzing the effect of this method on participants' reported implicit self-esteem, on their learning performance and affective states. Our methodology and experimental setup are described in the next section.

\section{METHOD}

\section{A. Environment}

The materials developed for this experiment consist of a multiple choice questionnaire related to logic. The questions are typically found in brain training exercises or in tests of reasoning ability. They involve inferential skills on information series and do not require particular prerequisites in any field of knowledge.
The questionnaire is composed of 3 modules. Each module is concerned with specific forms of data: the first module deals with geometrical shapes, the second module with numbers and the third module focuses on letters. In each module, learners have to answer to 5 multiple choice questions. The idea is to try to find the logical rule between the data, and guess the missing one.

Each Module starts with a tutorial giving instructions and examples to get learners accustomed with the user interface and types of questions. Learners are asked to respond as quickly and efficiently as possible to each of the 15 questions of the quiz. A correct answer was worth 4 points, an incorrect answer -1 , and a no-answer 0 .

\section{B. Enhancing Self-esteem}

In order to enhance learner self-esteem, we used an evaluative conditioning (EC) subliminal procedure [18]. This method consists in subliminally projecting selfreferent words (conditioned stimulus or CS) paired with positive words (unconditioned stimulus or US). The idea behind EC, is that conditioning influences the structure of associations in memory, and hence the automatic affective reactions resulting from these associations [18]. This method has already been found to influence self-esteem in earlier experiments (e.g. [10, 18]). Besides, it has been found that EC effects occur without awareness of the stimulus pairing.

Hence, in our experiment, some participants (experimental condition), were repeatedly presented with the subliminal primes (CS and US stimuli). The other participants (control condition), were not presented with subliminal primes. Projecting thresholds were carefully chosen according to neural recommendations [11]. Each subliminal prime (self-referent word and positive word) was displayed for $29 \mathrm{~ms}$ preceded and followed by a 271 ms mask of a set of sharp (\#) symbols. Figure 1 shows a diagram of the way subliminal priming took place.

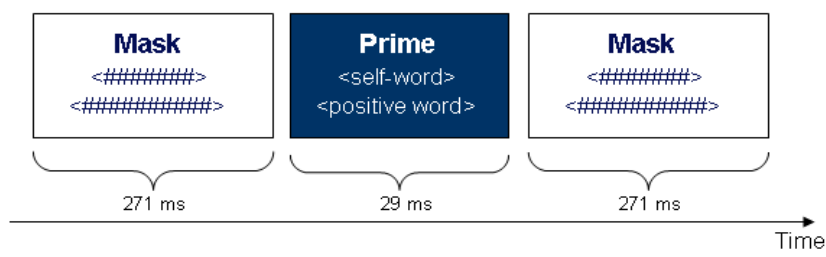

Figure 1. Subliminal priming of stimuli

Self-Esteem Measure: Learner self-esteem was assessed using the Initial Preference Task (IPT, [19]). Participants were asked to evaluate their attractiveness for all letters of the alphabet on a 7-point scale. Letters were presented individually, in random order on the screen. Participants pressed the corresponding key to evaluate each letter. High self-esteem is indexed by the extent to which a person prefers his or her initials to other letters of the alphabet. 


\section{Physiological Data Acquisition}

Physiological measures were recorded continuously during the experiment using a ProComp Infinity encoder. Three types of sensors were used: electroencephalogram (EEG), skin conductance (SC) and blood volume pulse (BVP) sensors. (1) EEG electrical brain activity was recorded using a Lycra stretch cap placed on the scalp. Cap electrodes were positioned according to the International 10/20 Electrode Placement System [20]. EEG signals were recorded from 4 scalp sites (P3, C3, Pz and $\mathrm{Fz}$ ). Each site was referred to $\mathrm{Cz}$ and grounded at Fpz. EEG signals were calibrated with regards to the average of left and right earlobe sites (A1 and A2). Each electrode site was filled with a small amount of electrolyte gel and sensor impedance was maintained below 5 kohms. The recorded sampling rate was at 256 Hz. (2) SC sensors were placed in the $2^{\text {nd }}$ and $4^{\text {th }}$ left hand finger. (3) BVP sensor was placed in the 3rd left hand finger. SC and BVP data were recorded at $1024 \mathrm{~Hz}$ of sampling rate. Heart rates (HR) were derived from BVP signals and galvanic skin response (GSR) from SC. All signals were notch filtered at $60 \mathrm{~Hz}$ to remove environmental interference during data acquisition.

Besides, two webcams were used to synchronize physiological signals with the tutoring system tasks. The former monitored the learner's facial activity and the latter recorded the learner's interactions on the computer screen.

Affect Recognition: From the physiological recorded signals, we wanted to analyze both learners' mental and emotional activities. In order to analyze the mental state, we used the recorded EEG signals. A Fast Fourier Transform (FFT) was applied to transform the signals into a power spectrum. The transformed signals were then divided into 3 frequency bands: theta $(4-8 \mathrm{~Hz})$, alpha (8$13 \mathrm{~Hz}$ ) and beta (13-22 Hz) [21]. Each of these wave types correlates with a particular mental state. More precisely, EEG literature on attention and vigilance [22] defined a mental engagement index given by:

$$
\text { engagement_index }=\frac{\text { combined_beta_power }}{\text { combined_alpha_power }+ \text { combined_theta_power }}
$$

We used this index as an indication of participant's mental engagement while answering to the questionnaire. The combined powers were sums of powers measured at electrode sites $\mathrm{P} 3, \mathrm{C} 3, \mathrm{Pz}$ and Fz. Higher index value reflects higher task engagement and alertness [21].

In order to assess learner emotional state, we used HR and GSR signals which are known to be correlated to valence (positive to negative) and arousal (low to high) [23]. It has also been established that emotions can be characterized in terms of valence and arousal [23]. Figure 2 depicts some named emotions in the arousal-valence space. In our analysis, we considered three levels for GSR signals and two levels for HR signals with regards to baseline. If GSR is $10-40 \%$ above the baseline, it is assumed as "high", for more than $40 \%$ it is considered as "very high" else it is assumed as "low". If HR is higher than baseline, it is assumed as "positive", else it is "negative". We then characterized learners' emotions as follows:

- "Relaxed" is defined by "low" GSR and "positive" HR.

- "Sad" is defined by "low" GSR and "negative" HR.

- "Joyful" is defined by "high" GSR and "positive" HR.

- "Frustrated" is defined by "high" GSR and "negative" HR.

- "Excited" is defined by "very high" GSR and "positive" HR.

- "Fear" is defined by "very high" GSR and "negative" HR.

We then focused on the proportion of emotions, i.e. we weighted the number of $\mathrm{HR}$ and GSR recordings corresponding to each of these emotion labels by the total number of recordings.

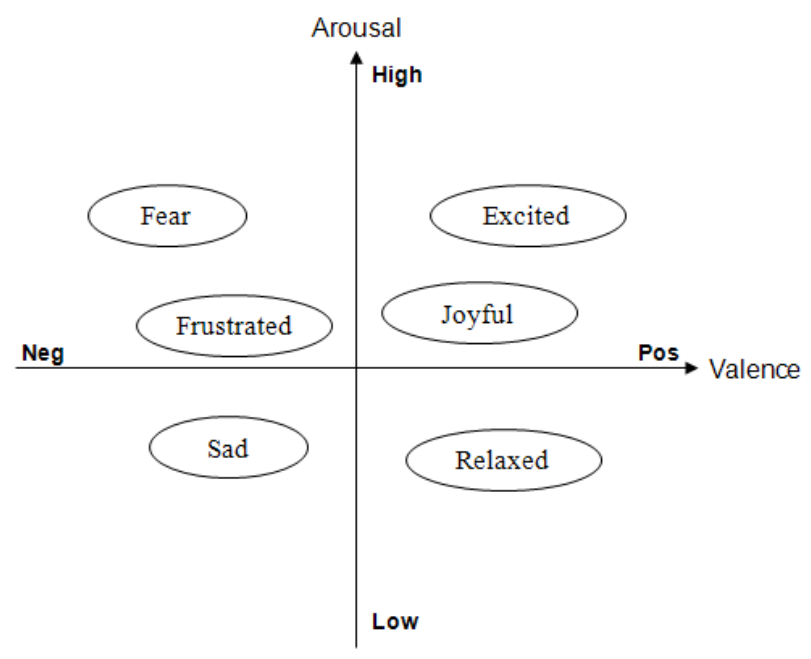

Figure 2. Some named emotions in the arousal-valence space

\section{Experiment Description}

Upon arrival at the laboratory participants were briefed about the procedure and consent was obtained. They were then randomly assigned either to the experimental condition or to the control condition. The former took place with self-esteem conditioning subliminal stimuli and the latter with no subliminal stimuli. Baselines for physiological signals were recorded during which participants were instructed to relax. The logic materials were then displayed with the instructions, warm-up examples and questions related to each of the three modules as described earlier. Finally, participants were asked to complete the IPT self-esteem scale.

\section{E. Participants}

39 participants ranged in age from 19 to 47 years $(M=$ $27.34, \mathrm{SD}=6.78$ ) took part to our study. They received 10 CAD for their participation. They were assigned either 
to the experimental condition (13 males, 7 females) or the control condition (11 males, 8 females).

\section{RESULTS AND DISCUSSION}

Results are presented in three sections. The first section presents self-esteem measure results. The second section deals with learner performance. Finally, the third section analyzes the learners' affective states.

Self-Esteem. Learner self-esteem was measured in terms of IPT effect by using the I-algorithm [19]. Mean rating of all non-initial letters is subtracted from each letter rating. Normative letter baselines are then computed by averaging the ipsatized letter ratings for individuals whose initials do not include the letter. The difference score is finally computed between the ipsatized initial ratings and the respective ipsatized baselines [19].

It was found that the computed self-esteem IPT effect was more pronounced for participants in the conditioned self-esteem (experimental) group $(\mathrm{M}=1.68, \mathrm{SD}=0.94)$ compared to participants in the control condition $(\mathrm{M}=$ $1.08, \mathrm{SD}=0.99$ ) indicating higher self-esteem. This difference was statistically reliable, $\mathrm{F}(1,37)=4.84$, $\mathrm{p}<$ 0.05 . Hence, results confirm that our method produced the expected main effect on learners' self-esteem.

Learning Performance. To measure learner performance we considered participants' final marks and time spent in the quiz. A main effect of priming condition was found for participants' final marks: they were significantly higher in the experimental condition $(\mathrm{M}=$ 33.4, $\mathrm{SD}=12.36$ ) compared to those in the control condition $(\mathrm{M}=25.5, \mathrm{SD}=9.87), \mathrm{F}(1,37)=4.37, \mathrm{p}<$ 0.05 . On the other hand, as depicted in Figure 3, it was found that in $1^{\text {st }}$ and $3^{\text {rd }}$ module, subliminally primed learners responded faster than the control group. A significant main effect was found for the $3^{\text {rd }}$ module $(\mathrm{F}(1$, $37)=4.545, \mathrm{p}<0.05)$ with $(\mathrm{M}=42.34, \mathrm{SD}=12.5)$ for the experimental condition and $(\mathrm{M}=51.67, \mathrm{SD}=13.7)$ for the control condition.

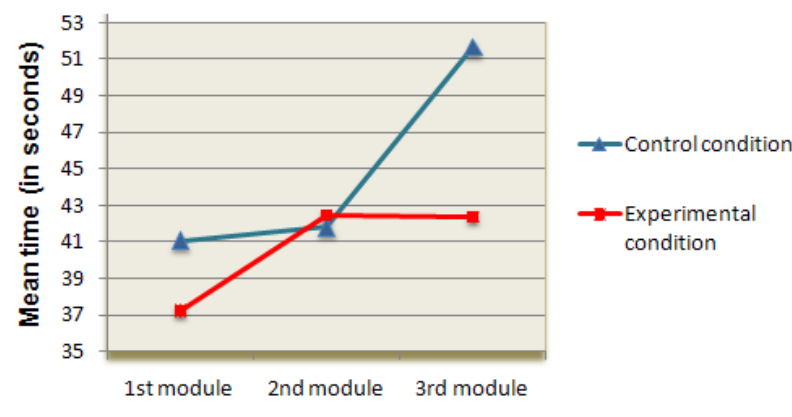

Figure 3. Mean time spent per module

To sum up these results, we found clear evidence of the positive effect of the priming strategy on learners' marks in the questionnaire. Response times seemed also to be enhanced; the effect was specifically more pronounced in the five last questions of the task.
Learner affect. Our next investigation was to compare mental and emotional activities between participants of the experimental condition and participants of the control condition. For the mental activity, we considered participants' mental engagement while answering to the questionnaire tasks.

Figure 4 sketches out the evolution of the mean engagement index in the questionnaire tasks for two participants. The first one was primed with subliminal self-esteem conditioning primes, and the second one wasn't projected with primes. It is shown that, in 9 questions over 15, the first participant has had a higher engagement index than the second one. A main overall effect was found: subliminally primed participants reported higher engagement index values $(\mathrm{M}=0.75, \mathrm{SD}=$ $0.11)$ than no primed participants $(\mathrm{M}=0.71, \mathrm{SD}=.09)$. $\mathrm{F}(1,583)=16.23, \mathrm{p}<0.001$.

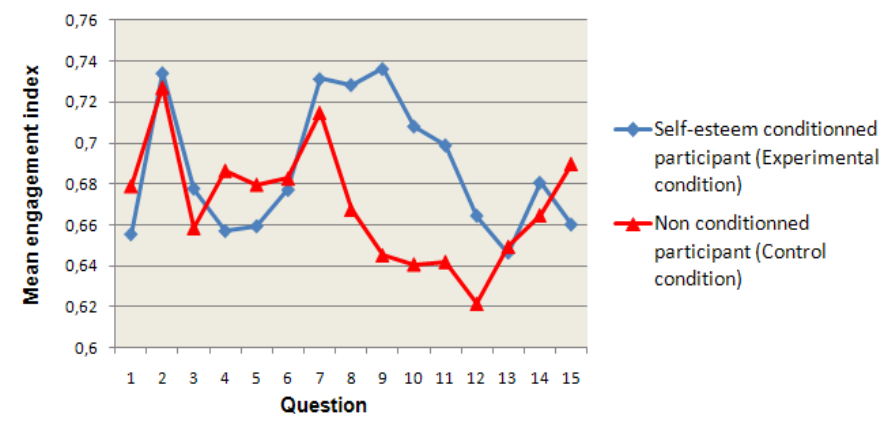

Figure 4. Mean engagement index evolution

To analyze participants' emotional states, we look at the proportions of emotions in each question of the quiz. A main effect of priming conditions was found for the emotions: "relaxed", "sad", "joyful", and "excited". ANOVA results and descriptive statistics are listed in Table 1. It was found that conditioned self-esteem participants have shown higher proportions of "joyful" and "excited" emotions described by a positive valence and a high to very high arousal and lower proportions of "relaxed" and "sad" emotions characterized by a low arousal level.

TABLE I. ANOVA RESULTS, MEAN AND STANDARD DEVIATION, OF EMOTION PROPORTIONS

\begin{tabular}{|l|c|c|c|c|c|}
\cline { 3 - 7 } \multicolumn{2}{c|}{} & \multicolumn{2}{c|}{$\begin{array}{c}\text { Experimental } \\
\text { condition }\end{array}$} & \multicolumn{2}{c|}{$\begin{array}{c}\text { Control } \\
\text { condition }\end{array}$} \\
\hline Emotions & $\boldsymbol{F}$ & $\boldsymbol{M}$ & $\boldsymbol{S D}$ & $\boldsymbol{M}$ & $\boldsymbol{S} \boldsymbol{D}$ \\
\hline Relaxed & $28.60^{\mathrm{a}}$ & 25.7 & 35.08 & 40.87 & 32.36 \\
\hline Sad & $77.73^{\mathrm{a}}$ & 10.02 & 18.13 & 26.76 & 26.77 \\
\hline Joyful & $45.00^{\mathrm{a}}$ & 38.27 & 35.92 & 14.94 & 23.84 \\
\hline Frustrated & 3.33 & 12.82 & 14.37 & 8.10 & 15.25 \\
\hline Excited & $0.59^{\mathrm{a}}$ & 4.88 & 15.55 & 2.48 & 8.84 \\
\hline Fear & 5.05 & 8.31 & 23.29 & 6.85 & 21.46 \\
\hline
\end{tabular}


From these results, it was found that priming conditions elicited different affective reactions among participants in terms of mental engagement index and emotions.

\section{CONCLUSION}

In this paper, we have proposed to enhance learner implicit self-esteem while interacting with a tutoring system. We used a subliminal self-esteem conditioning technique. An experiment was built to analyze the impact of this strategy on learning.

It was found that subliminally primed participants yielded higher learning performance in terms of marks obtained in the logic questionnaire. On the other side, self-esteem conditioning produced a positive effect on participants' mental engagement during tasks. It was also found that priming conditions elicited different emotional states with regards to valence and arousal activities. We believe that these findings can yield interesting implications in intelligent tutoring systems.

Our future work is directed towards studying the impact of this conditioning approach on a broader set of learner physiological features such as motivation and mental workload. We also plan to conduct deeper analysis on correlations between learner self-esteem level, emotions and mental state. In another perspective, we intend to model learners' level of self-esteem from their personal characteristics and physiological activities in order to extend the learner's module within an intelligent tutoring system.

\section{ACKNOWLEDGMENT}

We acknowledge the CRSNG (Conseil de Recherches en Sciences Naturelles et en Génie du Canada) and the Tunisian Government for their support.

\section{REFERENCES}

[1] R. Picard, Affective Computing: MIT Press, 1997.

[2] M. Chaouachi, A. Heraz, I. Jraidi, and C. Frasson, "Influence of Dominant Electrical Brainwaves on Learning Performance," in E-LEARN 2009, Vancouver, Canada, 2009.

[3] E. Jensen, "Brain-based learning: A reality check," Educational Leadership, vol. 57, pp. 76-80, 2000.

[4] S. D'Mello and A. Graesser, "Automatic detection of learner's affect from gross body language," Applied Artificial Intelligence, vol. 23, pp. 123-150, 2009.

[5] H. Prendinger and M. Ishizuka, "The empathic companion: A character-based interface that addresses users' affective states," Applied Artificial Intelligence, vol. 19, pp. 267-285, 2005.

[6] D. B. McFarlin and J. Blascovich, "Effects of selfesteem and performance feedback on future affective preferences and cognitive expectations," Journal of Personality and Social Psychology, vol. 40, pp. 521-531, 1981.
[7] B. C. Hansford and J. A. Hattie, " The Relationship between self and achievement/performance measures," Review of Educational Research, vol. 52, pp. 123-142, 1982.

S. W. McQuiggan, B. W. Mott, and J. C. Lester, "Modeling self-efficacy in intelligent tutoring systems: An inductive approach," User Modeling and User-Adapted Interaction, vol. 18, pp. 81-123, 2008.

[9] S. Harter, "Causes, correlates, and the functional role of global self-worth: A life-span perspective," in Competence considered, R. J. Sternberg and J. Kolligian, Eds. US: Yale University Press, 1990, pp. 67-97.

[10] A. P. Dijksterhuis, "I like myself but I don't know why: Enhancing implicit self-esteem by subliminal evaluative conditioning," Journal of Personality and Social Psychology, vol. 86, pp. 345-355, 2004.

[11] A. Del Cul, S. Baillet, and S. Dehaene, "Brain dynamics underlying the nonlinear threshold for access to consciousness," Public Library of Science, Biology, vol. 5, pp. 2408-2423, 2007.

[12] R. Hassin, J. Uleman, and J. Bargh, The new unconscious. Oxford, UK: Oxford University Press., 2005.

[13] F. L. Wallace, J. M. Flanery, and G. A. Knezek, "The effect of subliminal help presentations on learning a text editor," Inf. Process. Manage., vol. 27, pp. 211-218, 1991

[14] R. W. DeVaul, A. Pentland, and V. R. Corey, "The Memory Glasses: Subliminal vs.Overt Memory Support with Imperfect Information," Wearable Computers, IEEE International Symposium, 2003.

[15] P. Chalfoun and C. Frasson, "Subliminal priming enhances learning in a distant virtual 3D Intelligent Tutoring System," IEEE Multidisciplinary Engineering Education Magazine, vol. 3, pp. 125$130,2008$.

[16] R. F. Bornstein and P. R. D'Agostino, "Stimulus recognition and the mere exposure effect," Journal of Personality and Social Psychology, vol. 63, pp. 545-552, 1992.

[17] R. Radel, P. Sarrazin, P. Legrain, and L. Gobancé, "Subliminal Priming of Motivational Orientation in Educational Settings: Effect on Academic Performance Moderated by Mindfulness," Journal of Research in Personality, vol. 43, pp. 695-698, 2009.

[18] M. Grumm, S. Nestler, and G. v. Collani, "Changing explicit and implicit attitudes: The case of self-esteem," Journal of Experimental Social Psychology, vol. 45, pp. 327-335, 2009.

[19] E. P. LeBel and B. Gawronski, "How to find what's in a name: Scrutinizing the optimality of five scoring algorithms for the name-letter task," European Journal of Personality, vol. 23, pp. 85106, 2009.

[20] H. H. Jasper, "The ten-twenty electrode system of the International Federation," 
Electroencephalography and Clinical Neurophysiology, pp. 371-375, 1958.

[21] A. T. Pope, E. H. Bogart, and D. S. Bartolome, "Biocybernetic system evaluates indices of operator engagement in automated task," Biological Psychology, vol. 40, pp. 187-195, 1995.

[22] J. F. Lubar, "Discourse on the development of EEG diagnostics and biofeedback for attentiondeficit/hyperactivity disorders," Biofeedback and self-regulation, vol. 16, pp. 201-225, 1991.

[23] P. J. Lang, "The emotion probe: Studies of motivation and attention," American Psychologist, vol. 50, pp. 372-385, 1995. 